\title{
Detecting Basic Topological Changes in Sensor Networks by Local Aggregation
}

\author{
Jixiang Jiang \\ Spatial Information Science and Engineering \\ University of Maine \\ Orono, ME, USA \\ jixiang.jiang@maine.edu
}

\author{
Michael Worboys \\ Spatial Information Science and Engineering \\ University of Maine \\ Orono, ME, USA \\ worboys@spatial.maine.edu
}

\begin{abstract}
Wireless sensor networks (WSNs) can provide real-time information about geospatial environments, and so have the potential to play an important role in the monitoring of geographic phenomena. The research reported in this paper uses WSNs to provide salient information about spatially distributed dynamic fields, such as regional variations in temperature or concentration of a toxic gas. The focus is on topological changes to areas of high-activity that occur during the evolution of the field. Topological changes investigated include region merging and splitting, and hole formation or elimination. Such changes are formally characterized, and an algorithm is developed that detects such changes by means purely of in-network processing. The efficiency of this algorithm is investigated both theoretically and using simulation experiments.
\end{abstract}

\section{INTRODUCTION}

Wireless sensor networks provide real-time information about the environment, and thus have the potential to play an important role in the monitoring of geographic phenomena [13].

Geographical phenomena, such as wildfires or floods, can be abstracted to evolving areal objects. Taking a snapshot approach [6], at each moment in time an areal object can be generated as a union of regions selected according to certain criteria (e.g. locations where sensor readings are above a given threshold). The spatial evolution of the geographic phenomenon is represented as the evolution of these regions through time. In some applications, especially when areal objects are derived using discretization of continuous fields, the topological structure of the areal objects is complex, and changes to the topological structures are pervasive. We call the changes in topological structure of areal objects, topological changes.

Consider the three pairs of consecutive snapshots in Figure 1 , which are selected from a video showing the progression of the 1988 Yellowstone Park wild fire [19]. In the first pair of snapshots shown in Figure 1(a), a topological change of type '(fire zone) region appears' can be observed within the area enclosed by the bounding box A. In the second pair shown in Figure 1(b), a topological change of type 'regions merge' can be observed within the area enclosed by the bounding box $\mathrm{B}$, in which two fire zone regions become connected. In the third pair shown in Figure 1(c), a topological change of type 'region self-merges' can be observed within the area enclosed by the bounding box $\mathrm{C}$, in which a fire zone region merges with itself and forms a hole. These are examples of different types of topological changes. In addition, the inverses of these examples provide more examples of topological changes of types 'region disappears', 'region splits', 'region self-splits', respectively.

In many applications it is useful to have information about the kinds of topological changes. For example, in the case of wildfire, fire fighters might be interested if the fire zone regions split and become disconnected, so that they can reorganize the team accordingly. In the case of a flood, the emergency services may be interested in the appearance of an island in the flood, because this indicates the locations of possible safe areas. Instead of emerging from the flood directly, it is also possible that an island is formed by the flood engulfing a piece of land. In this scenario, people on the island become separated and may have difficulty escaping. Therefore, rescue from such a newly formed island might have higher priority in the overall hazard management strategy.

This work aims at detecting and reporting topological changes using sensor networks. Topological changes of areal objects are detected by the cooperation of sensors in the locality of the changes. Reports of the topological changes are generated and transmitted back to the base station as a collection of tuples of form $\left\langle t, x_{1}, y_{1}, x_{2}, y_{2}\right.$, TypeOfChange $\rangle$, where, $t$ is the time when a topological changes is detected, $\left(x_{1}, y_{1}\right)$ and $\left(x_{2}, y_{2}\right)$ form the lower-left and upper-right corners of a bounding box that exactly encloses the area of the observed topological change, and TypeOfChange indicates the type of observed topological change. The reports provide a general description of changes in particular areas. Users can then issue additional queries to the area that they are interested in to get more detailed information.

In order to enable such a reporting approach, we first provide a formal classification of different types of topological changes and point out the necessary information to distin- 


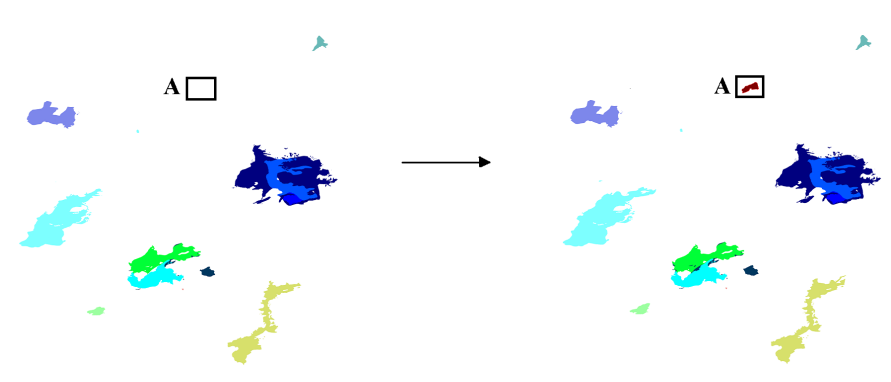

(a) Appear

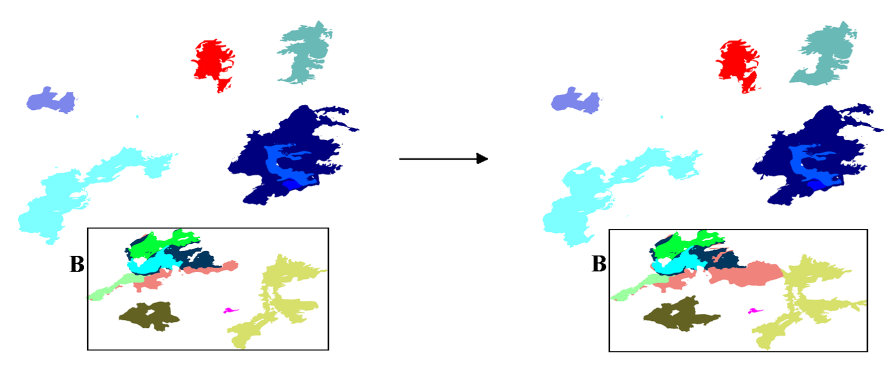

(b) Merge

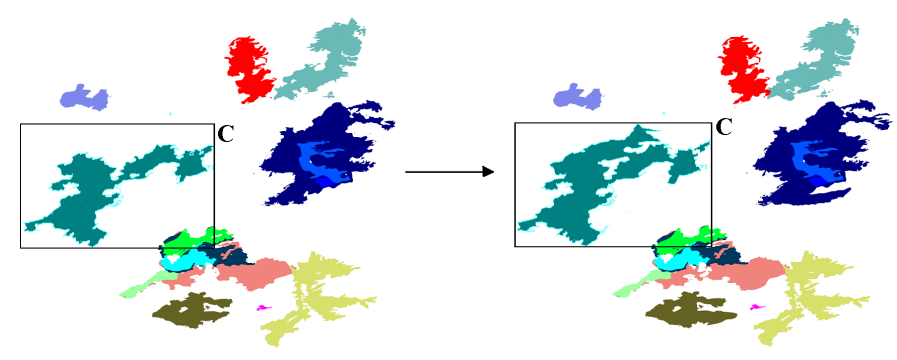

(c) Self-Merge

Figure 1: Examples of topological changes during the spreading of yellow stone wild fire

guish them. After that a distributed algorithm is proposed for the sensor networks to detect and report on the type of topological changes. Usually, to process topological information, especially to determine the connectivity of locations, requires cooperation between a large number of sensors and therefore is often energy consuming. In this work, the processing of topological changes is performed by a small proportion of sensors, which are identified dynamically after the change occurs. In this way, the communication cost can be reduced. As shown in the experiment, the total communication cost in our approach is much lower than the detection approach based on periodically reporting the boundaries of the areal objects to the sink.

\section{BACKGROUND}

This section surveys relevant research in topological models of areal objects, as well as topology detection in sensor networks.

Topological features are important aspects of areal objects, and representation of topological features is the focus of much research work in areal object modeling. Some modeling approaches support the representations of regions with one or more holes $[17,10,11]$. In addition to containing holes, an areal object may be unconnected (it is composed of one or more components), or weakly connected ( its boundary touches at a single or finite number of points). Examples of models representing areal objects with these richer topological properties include $[22,16]$.

Besides models representing the topology of static areal objects, current work has focused on the changes in topological structure of evolving areal objects. Jiang and Worboys [9] propose a model that represents the dynamic topology of areal objects, based on which different types of topological changes are specified. Meiden and Bronsvoort [21] analyze the changing topology affected by variations of feature parameters, and propose a method to determine the critical values of given variant parameters for which the topology changes.

Object tracking is one of the major applications of sensor networks. Previous work has focused on reducing the communication cost when transmitting the sensed data back to the base station. Recently, there is an increasing interest in considering topological information when processing sensed data. Gandhi, Hershberger and Suri [7] emphasize the topology of the isolines in a scalar field and propose an approach that approximates a family of isolines by a collection of topology-preserving polygons. Sarkar, Zhu and Gao etc. [24] present a distributed algorithm for the construction of a contour tree to represent the topology of a scalar field, based on which the isoline queries can be enabled.

In addition to the research on topology detection in static data, detection of topology in time-varying data with sensor networks is also considered. Worboys and Duckham [23] provide a computational model for sensor networks to detect global high-level topological changes based on lowlevel 'snapshots' of spatiotemporal data. Zhu, Sarkar, Gao, Mitchell [15] propose a distributed algorithm for sensor networks to maintain contours (or boundaries) of a binary object incrementally as they deform, while guaranteeing that the maintained contours capture the global topological features of the object boundary.

Another area of sensor network research related to the detection of areal object topology is the retrieval of network topology itself, especially the connectivity among sensor nodes in their communication graphs [12]. Deb, Bhatnagar and Nath [2] in their STREAM algorithm enable the retrieval of the entire network topology at a predefined resolution, which allows users to make a trade-off between topological details and resources expended. As holes in the sensor network are of primary interest in network management, some existing work detects sensor network holes using methods based on topological information [5], location information [3], or statistical information [4]. Although the detection of some topological properties of areal objects can be achieved based on the analysis of the network topology among sensors that 
observe the areal object [3], the cost for network topology detection is very high. Therefore, more efficient areal object topology detection methods are needed.

\section{CLASSIFICATION OF TOPOLOGICAL CHANGES}

In this section, we analyze the types of topological changes. In order to form the computational foundation for the detection and classification of topological changes, a formal description of various types of topological changes is provided.

Generally, an areal object at a particular time $t$ can be considered as a set of points $R$ in the spatial domain. As we are using areal objects to represent geographical phenomena at time $t, R$ is usually assumed to have finite number of regions, and each region has zero or a finite number of holes. Besides that, any regions or holes of an areal object is assumed to be purely two-dimensional; that is, they cannot be single points or line segments [16].

An areal object evolves through time. As the sensors usually take measurements at discrete times, the sensing reports are considered to be a sequence of snapshots of the areal object. Each pair of consecutive snapshots describes a change, called a basic transition. Topological changes can be observed during a basic transition. In the following discussion, we present the elements to distinguish different types of topological changes incured by a basic transition.

Let $R_{1}$ and $R_{2}$ be a pair of areal objects that define a basic transition, where $R_{1}$ and $R_{2}$ are the start and end snapshots, respectively. Any location $p$ in the spatial domain must have one of the following four states: (I) $p \notin R_{1}$ and $p \in R_{2}$. (II) $p \in R_{1}$ and $p \notin R_{2}$. (III) $p \in R_{1}$ and $p \in R_{2}$, or (IV) $p \notin R_{1}$ and $p \notin R_{2}$. Based on the states of each location, the whole spatial domain can be partitioned into several components. Each component is a maximal topologically connected set of locations in the same state. As an example, Figure 2(a) shows a basic transition of an areal object, and the spatial domain is indicated by the dashed-line box. The spatial domain is partitioned into components $a-g$ as shown in Figure 2 (b), among which, component $b$ consists of locations in state II. Components $a, d$, and $f$ consist of locations in state III. Components $c, e$, and $g$ consist of locations in state IV.

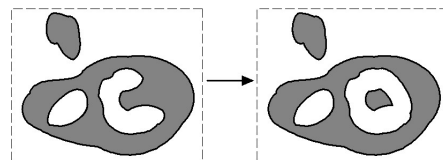

(a)

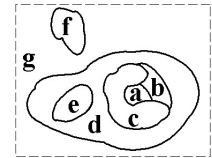

(b)

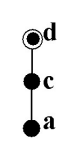

(c)
Figure 2: A basic transition

The locations in state I or state II form the components that are either added to or subtracted from the areal object during the basic transition. We call such components transition regions. In our current work, we assume each basic transition has only one transition region, and the transition region is a single piece without any holes. Both assumptions simplify our discussion on basic transitions. However, even with these assumptions, the detection of topological changes are still challenging. More complex transitions, in which more than one transition regions exist and each transition region is allowed to have holes, will be included in our future work. It should be pointed out that any complex transition can be decomposed into a sequence of basic transitions by first partitioning its transition regions into several simple transition regions, and then forcing the simple transition regions to switch one after another. Hence, the study of the basic transitions forms the foundation for the future work.

The locations with state III or IV form the components that do not change during the basic transition. Not all of them are crucial in the determination of the type of topological change. Only the components that are adjacent to the transition region are necessary. These components are referred to as $C$-components. In the example shown in Figure 2, only the Components $a, c$ and $d$ are C-components, as they are adjacent to the transition region $b$.

Similar to the discussion in binary images [14], the structure of the C-components in a basic transition have the properties stated as follows:

1. There is exactly one C-component $X$ which surrounds all the other C-components. $X$ is referred to as the background $C$-component of the basic transition.

2. The topological structure of the C-components in a basic transition can be represented by a rooted tree. A vertex of the tree represents a C-component, and an edge of the tree connects a pair of vertices representing adjacent $\mathrm{C}$-components. The root of the tree represents the background $\mathrm{C}$-component.

Back to the example shown in Figure 2(a), $d$ is the background C-component. In addition, $\mathrm{C}$-components $a$ and $c$ are adjacent, as well as the C-components $c$ and $d$. The structure of the $\mathrm{C}$-components can be represented by the rooted tree in Figure 2(c), in which the root is indicated by a double-circled vertex.

As different rooted tree can be explored in a systematic way, we are able to generate the possible topological structures between the C-components of a basic transition. Figure 3 lists all the rooted trees with less than 4 vertices, and examples of structures represented by the rooted trees are also provided. In the figure, the transition region is indicated by shaded area, and the vertices of the representation tree is placed inside the C-components they represent.
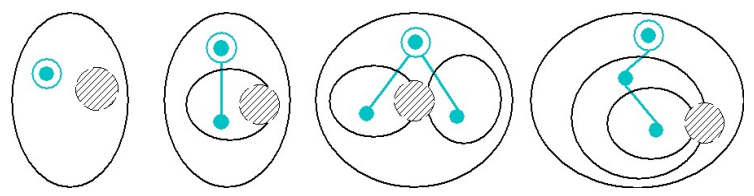

Figure 3: Tree representations for different configurations of $\mathrm{C}$-components

The classification of a basic transition is based on the following three factors: 
1. The topological structure of its C-components

2. The state of the locations in its transition region

3. The state of the locations in its background C-component

The classification yields different types of topological changes incurred by a basic transition. Figure 4 shows the classification results, in which the 13 types of specific topological changes are distinguished, and Figure 5 provides an example of a basic transition for each type of topological changes. These types of topological changes will be used in the sensor report to describe the observed basic transition.

\begin{tabular}{|c|c|c|c|c|}
\hline \multirow{2}{*}{\begin{tabular}{|l}
$\begin{array}{l}\text { Local } \\
\text { topological } \\
\text { structure }\end{array}$ \\
\end{tabular}} & \multicolumn{2}{|c|}{ State of transition region $(\mathrm{T})$} & \multicolumn{2}{|c|}{ State of background C-component (B) } \\
\hline & $T=I \quad B=I I I$ & $T=I I \quad B=I I I$ & $T=I \quad B=I V$ & $T=I I \quad B=I V$ \\
\hline 0 & Region appear (A) & Region disappear (B) & Hole disappear (I) & Hole appear (J) \\
\hline 0 & \multicolumn{4}{|c|}{ Topology-preserving changes $(\mathbf{C}, \mathbf{D}, \mathbf{K}, \mathbf{L})$} \\
\hline & Regions merge (E) & Region split (F) & Hole split (M) & Hole merge (N) \\
\hline & Region self-merge (G) & Region self-split (H) & Hole self-split (O) & Hole self-merge $(\mathbf{P})$ \\
\hline
\end{tabular}

Figure 4: Classification of basic transitions

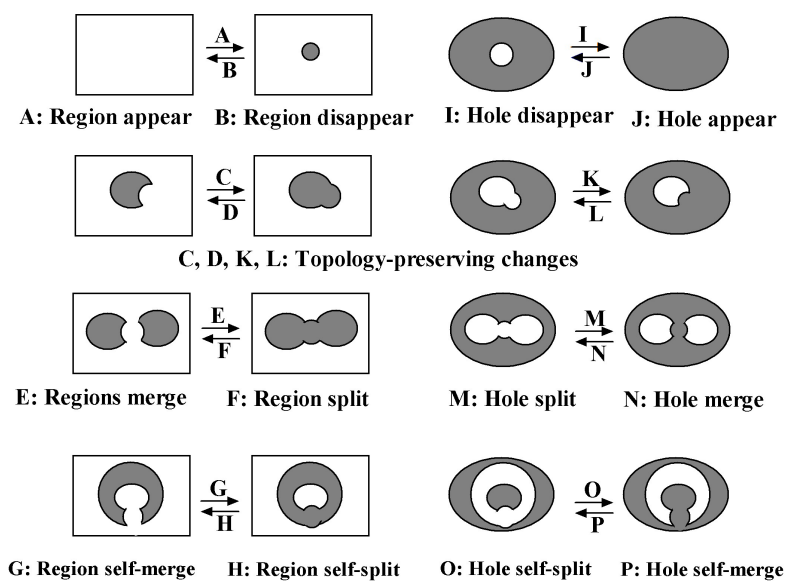

Figure 5: Examples of specific types of topological changes

It should be pointed out that in addition to the specific types of topological change, there are other topological changes whose topological structures are represented by a tree with four or even more vertices. Figure 6 gives an example of such changes and its representation tree. Such changes may not have a commonly accepted name associated with them, but they can also be detected and included in the sensing report if users are interested in them.

\section{TOPOLOGICAL CHANGE DETECTION}

This section presents an overview of the approach we propose for detecting and classifying topological changes in sensor networks, using the foundations laid in section 3 . In this work, we make the following assumptions of the sensors. Firstly, we assume for simplicity that the reading of a

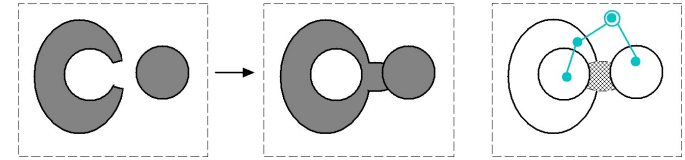

Figure 6: An example of uncommon topological change

sensor at any of the sampling times is either 0 or 1 . Our interpretation is that the reading is 1 if the sensor is in an area of high intensity, otherwise it is 0. Secondly, we assume that the sensors are densely deployed in the sensing area, and are able to communicate with their nearby neighbors. The density of the sensors is high enough to ensure that a pair of neighboring sensors with the same reading must be located in the same region or the same hole of the areal object, and a pair of neighboring sensors with different readings must be located on different sides of a boundary of the areal object. Finally, we assume that each sensor is initialized with a unique identifier and knows its location.

The sensors take measurements at each sensing round, and this leads to the determination of the type of the topological change. In order to determine the type of topological change, the sensors need to find the topological structure of the C-components, and to find the states of both the background $\mathrm{C}$-components and the transition region. In addition, a bounding box needs to be constructed to show the location of the observed topological change. All the necessary information can be collected by cooperation between sensor nodes located near the boundaries formed by different C-components and the transition region.

We use $r_{i, t} \in\{0,1\}$ to denote the reading of a sensor $n_{i}$ at time $t$, and use $t_{0}, t_{1}\left(t_{0}<t_{1}\right)$ to denote a pair of consecutive times at which sensors take their measurements. Colors of BLACK, WHITE and GRAY are first assigned to the nodes $n_{i}$ by comparing the readings of $r_{i, t_{0}}$ and $r_{i, t_{1}}$.

Definition 4.1. The color of node $n_{i}$ at time $t_{1}$ is defined to be:

$$
\begin{aligned}
& \text { 1. WHITE, if } r_{i, t_{0}}=r_{i, t_{1}}=0 \text {. } \\
& \text { 2. BLACK, if } r_{i, t_{0}}=r_{i, t_{1}}=1 \text {. } \\
& \text { 3. GRAY, otherwise. }
\end{aligned}
$$

By definition 4.1, all the GRAY nodes are located in the transition region. Some of the BLACK or WHITE nodes are located in the C-components, which are defined to be BLACK or WHITE C-component nodes nodes as follows:

Definition 4.2. A node $n$ is a BLACK C-component node, if $n$ is $B L A C K$, and $n$ is a 1-hop neighbor of either a GRAY node or another BLACK C-component node. The WHITE C-component node are defined in a similar way. Both types of nodes can be referred to as $\mathrm{C}$-component nodes. 
According to definition 4.2, a BLACK C-component node is a node that is connected to a GRAY node directly or by a sequence of nodes with the same reading. As the sensor nodes are densely deployed, the location of a BLACK C-component node must be in a component adjacent to the transition region. Therefore, a BLACK C-component node is located in a C-component. Similarly, a WHITE C-component is also located in a C-component.

Definition 4.3. A node $n$ is a BLUE node, if it is GRAY and has a k-hop neighbor of a BLACK or a WHITE node. $A$ node $n$ is a RED node, if it is a $C$-component node and has a $k$-hop neighbor that is either a $C$-component node with a different reading from $n$ or a GRAY node.

In definition $4.3, k \in\{1,2, \ldots\}$ is a parameter. $k$ is selected in order to ensure that the RED nodes in the same C-component form a connected component, and the BLUE nodes in the transition region form a connected component as well. As we have assumed the densely deployment of sensor nodes, $k$ is set to be 1 in our work. As an example, consider the basic transition described by Figures 7(a) and 7(b). A set of sensors are deployed to monitor the transition. The RED nodes identified after the transition are depicted by solid circles in Figure 7(b), and the BLUE nodes are depicted by solid squares.

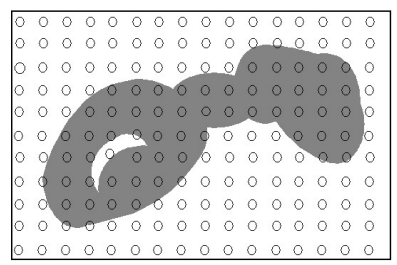

(a)

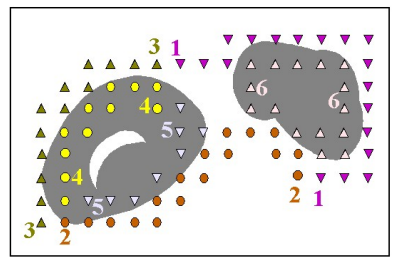

(c)

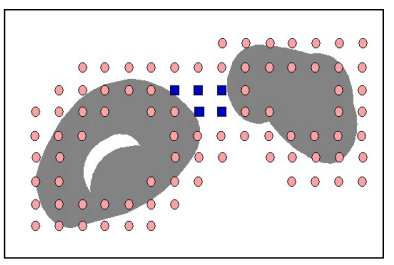

(b)

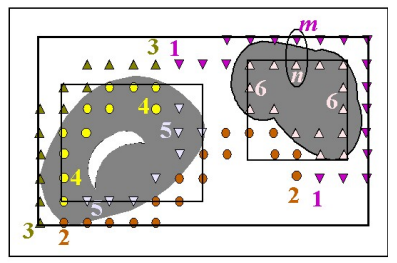

(d)
Figure 7: An example of node deployment

In order to collect information on the different C-components by the RED nodes, we need to set up the the relations between RED nodes and the C-component in which they are located. To do that, labels are assigned to the RED nodes. In this approach, the RED nodes located in the same Ccomponent form one or more clusters. The nodes hold the same label if, and only if, they belong to the same cluster. Based on the labels, each C-component can be named by the set of the labels assigned to the RED nodes located in it. The relationships between the nodes and C-component can be discovered according to their labels. A node with label $l$ is contained in the C-component named by the label set $L$, if and only if, $l \in L$. Figure 7 (c) shows a possible assignment of labels to the RED nodes in the example. The RED nodes form 6 clusters with labels 1-6, respectively. Nodes from each cluster are identified in Figure 7 (c) by a distinctive shape. Based on these labels, the three C-components of the transition can be named as $\{1,2,3\},\{4,5\}$, and $\{6\}$, respectively.

Based on the labeled clusters, the information for determining the type of topological changes is possible to be collected and represented. The necessary information to build the representation tree can be found by the RED nodes.

The adjacency relation between the C-components can be found by noting the neighboring RED nodes with different readings. If a pair of RED nodes labeled $l_{1}$ and $l_{2}$ are 1hop neighbors and have different readings, there must be a boundary formed by C-components that passes between them. Therefore, the C-components whose label sets contain labels $l_{1}$ and $l_{2}$ are adjacent. In the example, nodes $m$ and $n$ indicated in Figure $7(\mathrm{~d})$ are able to detect that the Ccomponents named $\{1,2,3\}$ and $\{6\}$ are adjacent. Other adjacency relation between different C-components can also be detected by RED nodes in a similar way.

The background C-component can be found using the bounding boxes of RED nodes. Each bounding box exactly encloses all the RED nodes located in a C-component. The bounding box of the RED nodes located in the background C-component must enclose the other bounding boxes, and therefore has the largest area. Based on this observation, the background C-component can be discovered by comparing the area of the bounding boxes. As an illustration, in Figure $7(\mathrm{~d})$ three bounding boxes are drawn, and each of them encloses the RED nodes in a C-component. Amongst the three bounding boxes, the one enclosing the RED nodes located in the background C-component named $\{1,2,3\}$ has the largest area.

The states of the background C-component as well as the transition region can be found by the RED and BLUE nodes located in them. With all the necessary information available, the type of change can be determined according to Figure 4. The result is contained as the TypeOfChange in the report. In addition, as the bounding box of the RED nodes located in the background C-component surrounds all the other C-components, it is included in the sensing report to describe the location of the observed topological change.

\section{ALGORITHM}

From the work of section 4, the necessary data to form the report to the base station can be gathered by individual BLUE and RED nodes. In this work, we use a local aggregation (LA) method to collect the data to determine the type of change. This method dynamically constructs a routing tree for data collection among the BLUE and RED nodes, and the detection procedure after each sensing round consists of the following three phases:

1. Diffusion phase, in which several tasks are done to prepare for data aggregation. The BLUE and RED nodes are identified. A special node, called the representative node, is selected among the BLUE nodes. The RED nodes form clusters, and nodes in each cluster hold a unique label. At the same time a routing 
tree is built, which connects all the RED nodes to the representative node.

2. Aggregation phase, in which the data necessary for detecting the topological changes from the RED nodes are transmitted back to the representative node along the routing tree.

3. Analysis phase, in which the representative node analyzes the data it has received and forms the report to be sent back to the base station.

Although the three phases are presented and will be described in a sequential order, in implementation the nodes are not required to be globally synchronized. Each node can start the next phase as long as it is confirmed to have finished the tasks in the previous phase. The following subsections describe each phase in detail.

\subsection{Diffusion phase}

In this phase, preparations are performed for the aggregation. After each sensing round each node can determine whether it is a WHITE, BLACK or GRAY node directly according to its local readings. However, the identification of BLUE and RED nodes needs the communication between the nodes. After a node confirms itself to be BLUE or RED, further communications are needed in order to form clusters, to assign labels, as well as to set up the routing structure. During this phase, the GRAY nodes have different behavior from the BLACK and WHITE nodes, and we describe them separately.

\subsubsection{Behavior of GRAY nodes}

In this phase the GRAY nodes communicate with their neighbors to identify the BLUE nodes. A representative node election procedure is performed among the identified BLUE nodes, after which, one of the BLUE nodes is elected to be the representative node. A by-product of the election is a routing tree, which connects all the BLUE nodes back to the representative node.

Assume a node $n$ discovers itself to be a GRAY node after a sensing takes place. Node $n$ first broadcasts a message $I S$ $G R A Y$ to its neighbors and waits. If $n$ discovers one of its neighbors to be a WHITE or BLACK node, $n$ is upgraded to a BLUE node, and enters the representative node election procedure.

The general idea of representative node election is that each BLUE node $n$ generates a random key value, and propagates it to its neighbors. The node with the smallest key value is selected to be the representative node. At the same time, each node selects the node from which the smallest key is first received as its parent, so that a routing tree is built that connects all the BLUE nodes to the representative node.

\subsubsection{Behavior of BLACK/WHITE nodes}

The first task of the BLACK/WHITE nodes in this phase is the identification of the RED nodes. According to definitions 4.2 and 4.3, a BLACK/WHITE node $n$ can be upgraded to a RED node if either of the following conditions is satisfied:
1. $n$ discovers a 1-hop neighboring GRAY node.

2. $n$ discovers a pair of 1-hop neighbors that are a BLACK C-component node and a WHITE C-component node.

As an example, after the transition described by Figures 7(a) and $7(\mathrm{~b})$ is observed, the RED nodes shown in Figure 8(a) are identified as satisfying condition 1 , and the RED node $n$ shown in Figure 8(b) is identified as satisfying condition 2.

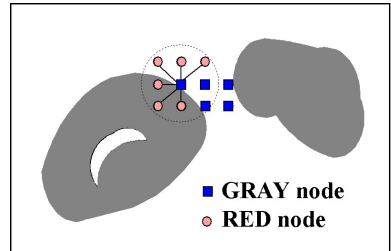

(a)

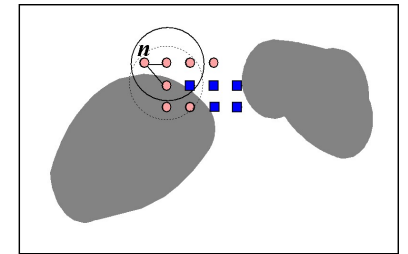

(b)
Figure 8: Examples of RED nodes identification

To confirm itself to be a RED node, a BLACK/WHITE node waits for messages from its neighbors after each sensing round. It is upgraded to a RED node if either condition is satisfied. If a BLACK/WHITE node $n$ receives a message indicating the existence of a 1-hop neighboring GRAY node (such as an IS-GRAY message from a 1-hop neighbor), using condition 1 , node $n$ is upgraded to a RED node directly, and after that broadcasts a message informing its neighbors about its upgrade. If a BLACK/WHITE node $n$ discovers a pair of 1-hop neighboring RED nodes upgraded one from a BLACK and one from a WHITE node, using condition 2, node $n$ is upgraded to a RED node. Node $n$ then broadcasts an upgrade message in order to continue identifying the other RED nodes.

After the RED nodes are identified, clusters are formed, and labels and routing structures are maintained amongst the RED nodes. In each C-component, some of the RED nodes that are identified using condition 1 are randomly selected to be the cluster heads. To form clusters, each cluster head initializes a flooding by propagating a cluster call message toward the other RED nodes located in the same Ccomponent. Each RED node joins with the same cluster as the sender of the cluster call message it first hears. During the cluster formation, all the RED nodes in the same cluster are assigned the same label, which is the unique identifier of its cluster head. Also a routing tree is set up within each cluster that is rooted at the cluster head and connects all the RED nodes in the cluster. Each cluster head is connected in turn to one of its neighboring BLUE nodes. As indicated in section 5.1.1, a routing tree is also formed amongst the BLUE nodes. Therefore, all the BLUE and RED nodes are connected in a single routing tree.

\subsection{Aggregation phase}

In this phase, the necessary data, including the adjacency relations between the clusters and a bounding box of the RED nodes in each cluster, are collected and transmitted back to the representative node. 
By communicating with its neighbors, a RED node observes the labels of its neighboring RED nodes. It then transmits this set of labels back to the cluster head along the routing tree. During the transmission, the same labels observed by different descendants are suppressed at their common ancestor. When the aggregation finishes, the cluster head knows the labels of its adjacent clusters.

With the routing tree built in each cluster, a bounding box that encloses all the RED nodes in a cluster can be built at the cluster head. The construction of the bounding box [20] is equivalent to finding, by a standard aggregation method, the minimum and the maximum $x$ and $y$ coordinates among all the RED nodes in that cluster.

Therefore, at the end of the aggregation phase, the head of each cluster $c$ is able to send to the representative node a package, including the label of $c$, the reading (either 0 or 1 ) of $c$, the bounding box enclosing the RED nodes in $c$, and the labels of clusters that are adjacent to $c$.

\subsection{Analysis phase}

After the aggregation phase, the data from all the clusters of RED nodes are transmitted to the representative node. In this phase, the representative node forms the report on the observed topological change based on the cluster level data.

In the analysis, clusters are formed into groups, each group having these properties:

1. All clusters in the same group have the same reading.

2. Clusters in the same group are connected with respect to the adjacency relation.

3. The group is maximal with respect to properties 1 and 2 .

Generally, each group satisfying these properties is formed by the clusters that are located in the same C-component. By generating all such groups of clusters, the C-components can be found, and each C-component can be represented by the set of cluster labels in a group.

The bounding box associated with a C-component can be constructed by fusing the bounding boxes of the clusters located in the $\mathrm{C}$-component. The construction of the bounding box is equivalent to finding the maximum and minimum coordinates of the bounding boxes associated with the clusters.

According to the analysis in section 4 , the adjacency relations between the C-components can be determined based on the the adjacency between the clusters. The background C-component can be found according to the bounding boxes associated with the C-components. The type of the locations in the transition region can be determined based on the local readings of the representative node. The type of the locations in the background C-component can be determined by the readings of the clusters located in it. Finally, the type of the topological change can be determined. A report is formed and sent back to the base station from the representative node.

\section{EXPERIMENT}

An experiment was conducted by simulation in order to test the performance of the proposed approach. We use Prowler [1], a MATLAB based network simulator, as our simulation environment.

\subsection{Experiment setup}

In the experiment, the size of the sensing area was set to be $420 \mathrm{~m} \times 600 \mathrm{~m}$, and sensors were deployed in a regular grid with the distance between adjacent sensors being $10 \mathrm{~m}$. Therefore, $42 \times 60=2460$ sensors in total were deployed. The sensing range of a sensor was defined to be $15 \mathrm{~m}$, so that each node is able to communicate with 8 neighbors. A sequence of 20 snapshots were generated from time $t_{1}$ to $t_{20}$, which described 19 basic transitions.

We first performed the topological detection by the proposed LA method, and different parameters were set up so that various numbers of RED node clusters were formed. A video was generated to show the state of the RED and BLUE nodes, as well as the routing tree built during the detection procedure. The video can be downloaded at [8]

For comparison, topological changes were also detected by the method of periodically boundary reporting, a basic spatial suppression (SS) approach. This method has been widely used as a comparison [24, 18]. In the SS approach, after each sensing round sensors first communicated with their neighbors to identify the boundary nodes, or the nodes that had a neighboring node with a different reading. After that, the boundary nodes located in the areal object reported their IDs back to the base station via the shortest routes. We assumed that the the base station knew the location of each sensor. With the IDs received after each sensing round, the base station was able to generate the boundary of the areal object. As the boundary characterized the topological properties of an areal object, the topological changes could also be identified based on the consecutive snapshots of the boundary.

\subsection{Results and discussion}

In the experiments, all the reported types of topological changes by both methods were the same as expected.

Table 1 lists the information related to both methods after each sensing round. For the LA method, it presents the number of RED nodes being identified (No. of RED Nodes), the total number of bytes transmitted during the diffusion phase (Cost in Diff.) and during the aggregation phase (Cost in Agg.). For the boundary reporting method, it presents the number of boundary nodes located in the areal object (No. of Bd Nodes), and the total number of bytes transmitted (Cost). Generally, the LA method has lower communication than the SS method.

We first consider a typical sensing round, and analyze the difference between the two approaches. Figure 9(a) shows a basic transition described by the snapshots of areal objects at $t_{15}$ and $t_{16}$. By the LA method, at time $t_{16}$, the nodes 
Table 1: Simulation results

\begin{tabular}{|c|c|c|c|c|c|c|}
\hline & \multicolumn{2}{|c|}{ By Local Aggregation } & \multicolumn{2}{c|}{ By Boundary Reporting } \\
\hline Time & Type of change & No. of RED Nodes & $\begin{array}{c}\text { Cost in Diff. } \\
\text { (bytes) }\end{array}$ & $\begin{array}{c}\text { Cost in Agg. } \\
\text { (bytes) }\end{array}$ & No. of Bd Nodes & $\begin{array}{c}\text { Cost } \\
\text { (bytes) }\end{array}$ \\
\hline$t_{0}$ & & 0 & 0 & 0 & 86 & 6664 \\
\hline$t_{1}$ & Topology-preserving & 170 & 1447 & 1210 & 97 & 7186 \\
\hline$t_{2}$ & Region Appear & 64 & 668 & 1327 & 129 & 9796 \\
\hline$t_{3}$ & Region Merge & 284 & 2338 & 2428 & 142 & 10826 \\
\hline$t_{4}$ & Topology-preserving & 336 & 2710 & 2465 & 150 & 11218 \\
\hline$t_{5}$ & Region Merge & 536 & 4458 & 3364 & 146 & 10984 \\
\hline$t_{6}$ & Topology-preserving & 576 & 4832 & 2865 & 159 & 11818 \\
\hline$t_{7}$ & Topology-preserving & 606 & 5050 & 3027 & 165 & 12158 \\
\hline$t_{8}$ & Region Split & 612 & 4974 & 3787 & 171 & 12512 \\
\hline$t_{9}$ & Topology-preserving & 228 & 1985 & 2102 & 193 & 14130 \\
\hline$t_{10}$ & Region Split & 414 & 3386 & 2676 & 187 & 13582 \\
\hline$t_{11}$ & Region Merge & 584 & 4710 & 3256 & 197 & 14124 \\
\hline$t_{12}$ & Topology-preserving & 616 & 4867 & 3471 & 194 & 13886 \\
\hline$t_{13}$ & Topology-preserving & 638 & 5075 & 3848 & 202 & 14346 \\
\hline$t_{14}$ & Region self-merge & 622 & 4844 & 3450 & 194 & 13920 \\
\hline$t_{15}$ & Topology-preserving & 530 & 4449 & 2554 & 192 & 13800 \\
\hline$t_{16}$ & Hole disappear & 60 & 638 & 909 & 170 & 12538 \\
\hline$t_{17}$ & Region appear & 52 & 544 & 798 & 196 & 14668 \\
\hline$t_{18}$ & Region Merge & 588 & 4661 & 3246 & 189 & 14132 \\
\hline$t_{19}$ & Topology-preserving & 696 & 5886 & 4058 & 190 & 14224 \\
\hline Total communication cost & & 117156 bytes & & 246512 bytes \\
\hline
\end{tabular}

participating in the data aggregation are shown in Figure 9(b), in which the solid circles represent RED nodes, and the hollow squares represent the BLUE nodes. The report on topological change is first formed by the communication amongst the RED and BLUE nodes, and a unique representative node sends the final report back to the base station located at the up-left corner of the sensing area. 1342 bytes transmission in total are needed. For the SS method, at time $t_{16}$ the boundary nodes located in the areal object are shown in Figure 9(c). Each boundary node sends its ID back to the base station, and Figure 9(c) shows the paths for the data transmission of two boundary nodes. As there are 196 boundary nodes, 14668 bytes transmission are needed for the reporting.

In the LA method, the data is first transmitted and aggregated among the RED and BLUE nodes locally, and after that a representative node transmits a single data package back to the base station. In the SS method, each boundary node needs to send its identifier back to the base station. As sending the data back to the base station costs more than sending the data back to the local representative node, the LA method outperforms the SS method. In this experiment, the total communication cost in detecting the 19 topological changes by the LA method is 116956 bytes, which is less than half of the communication cost by the SS method (246512 bytes).

The communication cost in the LA method is also affected by several factors, among which, the number of RED nodes is the most important. In order to further study the effect of the number of RED nodes on the communication cost, we plotted the average communication cost in a sensing round against the average number of RED nodes, as shown in Figure 10. Generally, the communication cost in both the diffusion phase and the aggregation phase increases almost linearly with the number of RED nodes identified.

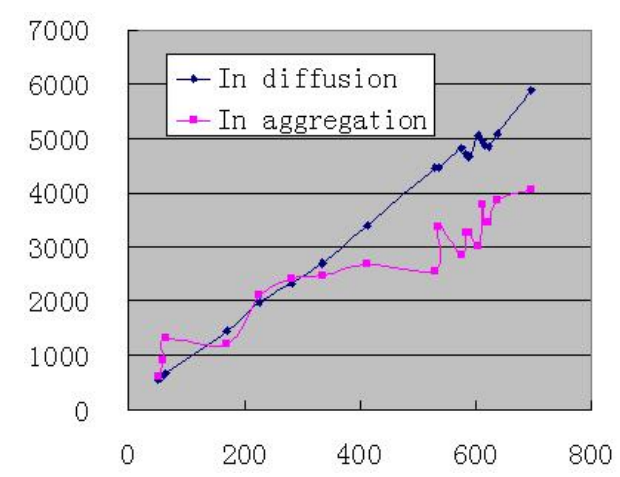

Figure 10: Communication cost against the number of RED nodes

Given the same number of RED nodes, the number of clusters formed among the RED nodes also affected the communication cost. To study this effect, we considered the relation between total communication cost and the total number of clusters formed in detecting the 19 topological changes. We performed the experiment 6 times, and each time different number of clusters were formed. Figure 11 plots the total number of bytes being transmitted against the total number of clusters formed. Generally, the communication cost in diffusion phase remains stable and is not affected by the number of clusters. However, the communication cost in aggregation phase increases with the increase of number of clusters. 


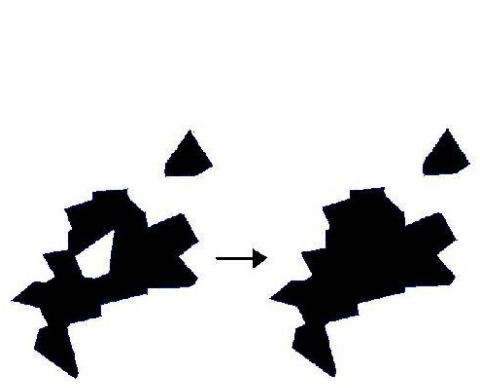

(a)

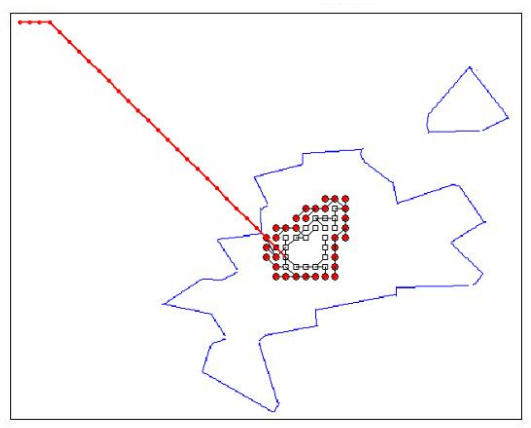

(b)

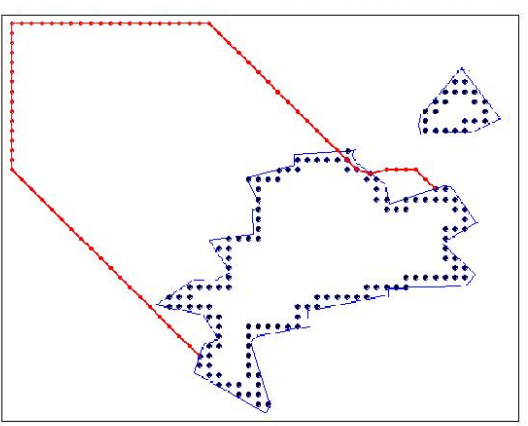

(c)

Figure 9: The difference of both approaches in data collecting

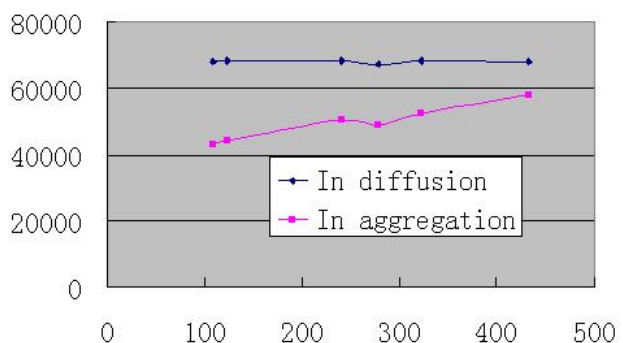

Figure 11: Communication cost against the number of clusters

\section{CONCLUSIONS AND FUTURE WORK}

This paper gives a treatment of the topological changes incurred by a basic transition. We classify a basic transition according to its local topological structure as well as the state of the transition region and its neighborhood.

A distributed algorithm is proposed, which enables densely deployed sensor network to detect different types of topological changes and report to the base station. We have shown that the detection process can be completed by the cooperation of sensors that observe the boundary of the Ccomponents. These nodes form clusters, and the clusterlevel data can be gathered to determine the type of topological changes. As shown by the simulation results, our approach requires only half of the communication cost that is required by the approach using periodical boundary construction.

In the current approach, a cluster of RED nodes exists only in one sensing round. However, many RED nodes may participate in the detection of topological changes for more than one consecutive sensing rounds. Therefore, there may be redundancy in communication during the repeated formation of clusters among the same set of nodes. So future work will develop a new approach that reuses the cluster data over time. In the projected approach, the nodes located near the boundary of the areal object form clusters, and the cluster data is maintained at the base station. After each sensing round, only the clusters located near the transition region are updated, and the reports are sent to the base station from these updated clusters. In this way, the base station is able to detect the type of change based on the cluster data. We hope this will lead to more efficient topological change detection. In addition, the new approach enables us to process more than one transition region at the same time, and therefore may also provide a way to deal with complex transitions, in which more than one transition regions exist.

\section{REFERENCES}

[1] Prowler-probabilistic wireless network simulator. http://www.isis.vanderbilt.edu/Projects/nest/ prowler/. (Last accessed June 4, 2008).

[2] B. Deb, S. Bhatnagar, and B. Nath. STREAM: Sensor topology retrieval at multiple resolutions. Telecommunication Systems, 26(2-4):285-320, 2004.

[3] Q. Fang, J. Gao, and L. Guibas. Locating and bypassing routing holes in sensor networks. In Mobile Networks and Applications, volume 11, pages 187-200, 2006.

[4] S. Fekete, A. Kröller, D. Pfisterer, S. Fischer, and C. Buschmann. Neighborhood-based topology recognition in sensor networks. In Algorithmic Aspects of Wireless Sensor Networks, LNCS 3121, pages 123-136, 2004.

[5] S. Funke. Topological hole detection in wireless sensor networks and its applications. In Proceedings of the 2005 Joint Workshop on Foundations of Mobile Computing, pages 44-53, Cologne, Germany, 2005.

[6] A. Galton. Fields and objects in space, time, and space-time. Spatial Cognition and Computation, 4(1):39-68, 2004

[7] S. Gandhi, J. Hershberger, and S. Suri. Approximate isocontours and spatial summaries for sensor networks. In Proceedings of the 6th International Symposium on Information Processing in Sensor Networks (IPSN'07), pages 400-409, Cambridge, MA, USA, 2007.

[8] J. Jiang. Experiment video of topological change detection. http:

//www.spatial.maine.edu/ ${ }^{j j i a n g / t p c h a n g e s . z i p, ~}$ 2008. (Last accessed June. 6, 2008).

[9] J. Jiang and M. Worboys. Specifying events by changes in topological properties (extended abstract). In 4th International Conference on Geographic 
Information Science (GIScience 2006), 2006.

[10] A. Kilgour. Techniques for modeling and displaying 3D scenes. Advances in Computer Graphics II, pages $55-113,1986$

[11] W. Kropatsch. Building irregulars pyramids by dual graph contraction. IEE Proceedings on Vision, Image and Signal Processing, 142(6):366-374, 1995.

[12] M. Li and B. Yang. A survey on topology issues in wireless sensor network. In Proceedings of the 2006 International Conference on Wireless Networks, page 503, Las Vegas, Nevada, USA, 2006.

[13] S. Nittel, A. Stefanidis, I. Cruz, M. Egenhofer, D. Goldin, A. Howard, A. Labrinidis, S. Madden, A. Voisard, and M. Worboys. Report from the First Workshop on Geo Sensor Networks. ACM SIGMOD Record, 33(1):141-144, 2004

[14] A. Rosenfeld. Adjacency in digital pictures. Information and Control, 26:24-33, 1974.

[15] R. Sarkar, X. Zhu, J. Gao, L. Guibas, and J. Mitchell. Iso-contour queries and gradient descent with guaranteed delivery in sensor networks. In Proceedings of the 27th Annual IEEE Conference on Computer Communications (INFOCOM'08), Phoenix, AZ, USA, 2008.

[16] M. Schneider and T. Behr. Topological relationships between complex spatial objects. ACM Transactions on Databases Systems, 31(1):39-81, 2006.

[17] M. Scholl and A. Voisard. Object-oriented database systems for geographic applications: an experiment with $\mathrm{O}_{2}$. In F. Bancilhon, C. Delobel, and P. C. Kanellakis, editors, The $\mathrm{O}_{2}$ Book, pages 585-618. Morgan Kaufmann, 1992.

[18] A. Silberstein, R. Braynard, and J. Yang. Constraint chaining: on energy-efficient continuous monitoring in sensor networks. In Proceedings of the 2006 ACM SIGMOD International Conference on Management of Data (SIGMOD '06), pages 157-168, 2006.

[19] E. Sokolowsky, H. Mitchell, and S. Maher. Wildfire growth around Yellowstone National Park in 1988 (WMS). http://svs.gsfc.nasa.gov/vis/a000000/ a002900/a002909/index.html, 2004. (Last accessed Nov. 30, 2007).

[20] N. Trigoni, Y. Yao, A. Demers, J. Gehrke, and R. Rajaraman. Multi-query optimization for sensor networks (DCOSS'05). In Proceedings of International Conference on Distributed Computing in Sensor Systems, pages 307-32, 2005.

[21] H. van der Meiden and W. Bronsvoort. Tracking topological changes in feature models. In Proceedings of the 2007 ACM Symposium on Solid and Physical Modeling, pages 341-346, Beijing, China, 2007.

[22] M. Worboys and P. Bofakos. A canonical model for a class of areal spatial data. In Proceedings of the 3rd International Symposium, SSD'93, LNCS 692, pages 36-52, 1993.

[23] M. Worboys and M. Duckham. Monitoring qualitative spatiotemporal change for geosensor networks. International Journal of Geographical Information Science, 20(10):1087-1108, 2006.

[24] X. Zhu, R. Sarkar, J. Gao, and J. Mitchell. Light-weight contour tracking in wireless sensor networks. In Proceedings of the 27th Annual IEEE
Conference on Computer Communications (INFOCOM'08), Phoenix, AZ, USA, 2008. 